\title{
Introductory Lectures on Topological Quantum Field Theory
}

\section{Wolfger Peelaers*}

Mathematical Institute, University of Oxford, Woodstock Road, Oxford, OX2 6GG, UK

E-mail: wolfger.peelaers@maths.ox.ac.uk

\begin{abstract}
Topological quantum field theory (TQFT) is a vast and rich subject that relates in a profound manner physical observables to topological invariants. These lecture notes provide an elementary introduction to the subject. We will introduce Atiyah's axiomatic definition of topological quantum field theories and explain how it provides a particularly intuitive, pictorial representation of the algebraic structure of two-dimensional TQFTs. We also consider Witten's topological twist as a means to obtain so-called cohomological TQFTs. These notes are based on lectures given at the XV Modave Summer School in Mathematical Physics.
\end{abstract}

XV Modave Summer School in Mathematical Physics - Modave2019

8-14 September 2019

Modave, Belgium

${ }^{*}$ Speaker. 


\section{Introduction}

Topological quantum field theories are characterized by the property that their observables only depend on the global characteristics of the space on which they are defined. In other words, their observables compute topological invariants of said space, and thus, in particular, don't depend on the metric. As any quantum field theory, a TQFT can be studied in a Hamiltonian framework or using its Lagrangian description and the path integral. The former approach is characterized by a trivial Hamiltonian $H=0$. Nevertheless, nontrivial propagation does occur through topologically nontrivial spacetimes. This approach was axiomatized in [1] and is the subject of the first part of these lecture notes.

In the path integral approach to topological quantum field theory two distinct mechanisms have been developed to ensure that observables are independent of the metric. The first one, not covered in these lectures, insists on that neither the action nor the observables have any explicit metric dependence. Such topological quantum field theories are called of Schwarz-type, after the author of the paper [2]. An important example of this type is three-dimensional Chern-Simons theory, which facilitates the computation of invariants of three-manifold and knots embedded therein [3]. The second mechanism is cohomological in nature: only after passing to the cohomology of a particular nilpotent charge does the metric dependence disappear. Topological twisting provides a powerful and widely applicable method to construct quantum field theories with a suitable nilpotent charge [4]. The second part of these lecture notes studies this setup.

These notes do not intend to be original or comprehensive. For example, we have omitted the rephrasing of the axiomatic definition of a topological quantum field theory in categorical language, and have barely scratched the surface of cohomological quantum field theory and the computation of its topological observables. Instead, these lectures aim to give a pedestrian introduction to the above-mentioned topics. Many lecture notes, reviews, and text books are readily available, for example $[5,6,7,8,9]$, for the interested reader to continue their study of topological quantum field theory.

These notes are organized as follows. In section 2 we briefly review some aspects of topology and introduce cobordisms. In section 3, we develop the axiomatic approach to topological quantum field theory, focusing in particular on two-dimensional theories. This section is largely based on the excellent text book [8]. Finally, section 4 introduces cohomological topological quantum field theories and explores Witten's topological twisting, mostly following the seminal paper [4].

\section{Topological properties and cobordisms}

A topological quantum field theory is a quantum field theory defined on a topological space whose observables compute topological properties of that space. Let us start by explaining the mathematical ingredients of this definition.

\subsection{Topological invariants}

Mathematically, a topological space is an ordered pair $(X, \tau)$, with $X$ a set and $\tau$ a collection of subsets of $X$, such that i) $\emptyset$ and $X$ belong to $\tau$, ii) any arbitrary (finite or infinite) union of elements of $\tau$ belongs to $\tau$, and iii) the intersection of any finite number of elements of $\tau$ belongs 
to $\tau$. The elements of $\tau$ are called open sets, while $\tau$ itself goes under the name of a topology on $X$. Intuitively, a topology tells us which points of $X$ lie "close" or "in the neighborhood" of each other (without caring about any precise concept of distance). Indeed, the notion of a neighborhood formalizes this intuition. A subset $N \subseteq X$ is a neighborhood of a point $x \in X$ if there exists an open set $U \in \tau$ such that $x \in U$ and $U \subseteq N$. A few examples of topological spaces are as follows:

- for $X$ any set, we can set $\tau$ equal to the collection of all subsets of $X$. This is called the discrete topology.

- for $X$ any set, we can take $\tau=\{\emptyset, X\}$. This is the so-called trivial topology.

- a simple example of a topological space that is neither discrete nor trivial is the Sierpinski space. It is based on the set $X=\{0,1\}$ and chooses $\tau=\{\emptyset,\{0,1\},\{1\}\}$.

- for applications in physics, the set $X$ is most often a metric space and thus possesses a metric specifying the distance between points. We can use the metric to endow such space with a metric topology. The basic open sets are open balls defined by the metric and $\tau$ comprises all their possible unions.

To introduce topological properties or invariants, we first define a function $f: X \rightarrow Y$ between the topological spaces $\left(X, \tau_{X}\right)$ and $\left(Y, \tau_{Y}\right)$ to be continuous if for all $x \in X$ and all neighborhoods $N$ of $f(x)$, there exists a neighborhood $M$ of $x$ such that $f(M) \subseteq N .{ }^{1}$ A homeomorphism $f$ is a bijection with both $f$ and $f^{-1}$ continuous. Two topological spaces with a homeomorphism between them are essentially identical from the standpoint of topology. Finally, a topological property is a property of a topological space that is invariant under homeomorphisms. For metric spaces with the metric topology, roughly, a topological property is thus a property that does not depend on the choice of metric.

\subsubsection{Classification of connected, compact, topological surfaces}

For orientable and closed surfaces a complete classification of topological surfaces is as follows. ${ }^{2}$ Either it is the sphere or it is the connected sum of $g$ tori for $g \geq 0$. The topological property that tells these spaces apart is the number of holes $g$, also known as the genus. One often also considers the Euler characteristic $\chi=2-2 g$.

For orientable surfaces with boundary, the above classification is extended by including the count of the (finite) number $k$ of windows/punctures. The Euler characteristic is now computed as $\chi=2-2 g-k$. In fact, since the surface is orientable, we can keep track of the number of inand out-boundaries, $k_{\text {in }}$ and $k_{\text {out }}$, separately. We denote a genus $g$ surface with $k_{\text {in/out }}$ boundaries as $\Sigma_{g ; k_{\text {in }}, k_{\text {out }}}$.

Unorientable surfaces are classified topologically by their genus, their number of windows, and their number of cross-caps $c$. The latter can take the values $c=0,1,2$. For example, a sphere with a cross-cap is the real projective plane, while a sphere with two cross-caps is the one-sided

\footnotetext{
${ }^{1}$ Note the analogy to the standard $\varepsilon-\delta$ definition of continuity first encountered in high school for real functions $f: \mathbb{R} \rightarrow \mathbb{R}$.

${ }^{2} \mathrm{~A}$ closed surfaces is a compact surface without boundary. The intuitive notion of compactness of $(X, \tau)$ can be formalized in topology by stating that any open cover of $X$ has a finite subcover.
} 


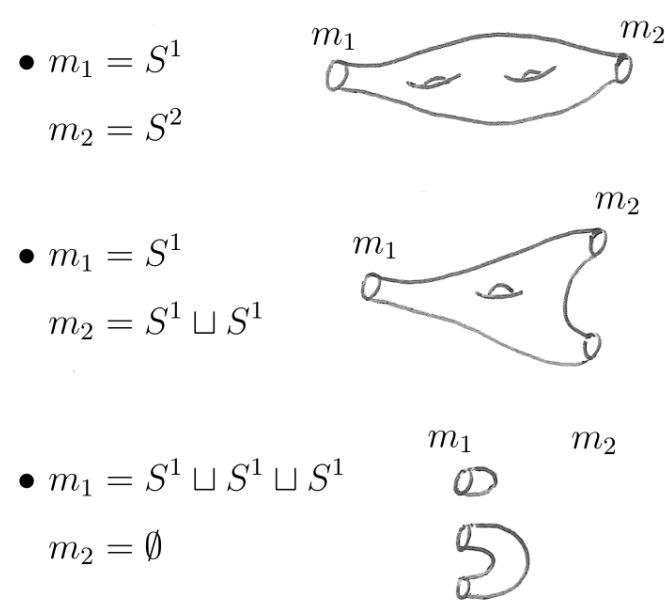

Figure 1: Simple examples of cobordisms $M$ between $m_{1}$ and $m_{2}$.

surface of the Klein bottle. The Euler characteristic reads $\chi=2-2 g-c-k$. Note that if $c \geq 3$, one can replace pairs of cross-caps by handles.

\subsection{Cobordisms}

Cobordisms lie at the heart of the Hamiltonian picture of topological quantum field theory. They are defined as follows. Consider two closed manifolds $m_{1}, m_{2}$ of dimension $n-1$. A cobordism between $m_{1}$ and $m_{2}$ is then a compact $n$-manifold $M$ whose boundary is (homeomorphic to) $m_{1} \sqcup m_{2}$. See figure 1 for some two-dimensional examples.

Not all pairs of $(n-1)$-dimensional manifolds are cobordant to each other. For example, any two closed one-manifolds are cobordant, but two closed 0-manifolds (a disjoint union of points) are cobordant if and only if the total number of points is even.

For the purposes of these lectures, we will restrict attention to oriented cobordisms. An oriented cobordism between two closed, oriented manifolds $m_{1}, m_{2}$ of dimension $n-1$ is a compact, oriented $n$-manifold whose in-boundary is (homeomorphic to) $m_{1}$ and whose out-boundary is (homeomorphic to) $m_{2}$. (We will always be working up to diffeomorphisms and henceforth won't explicitly make the "up to homeomorphisms" disclaimer anymore.) We denote an oriented cobordism as $M: m_{1} \Rightarrow m_{2}$.

If $m_{1}$ and $m_{2}$ are homeomorphic, then an important cobordism is the cylinder:

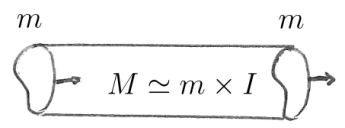

A second important cobordism is the $U$-tube. Consider $m$ and $\bar{m}$, i.e., $m$ with opposite orientation. We can consider the cylinder $m \Rightarrow \bar{m}$, but as both boundaries are in-boundaries and the out-boundary is empty, it is customary to represent it as in figure 2a. Similar statements hold for the cylinder $\bar{m} \Rightarrow m$. 


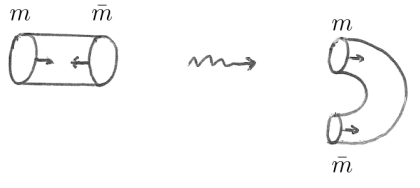

(a)

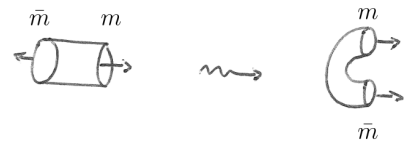

(b)

Figure 2: U-tube cobordisms.
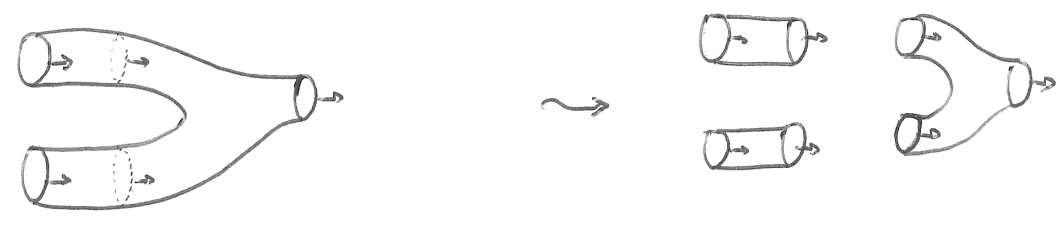

Figure 3: Example decomposition of a cobordism.

Note that if $n=2$, the topological surface $\Sigma_{g, k_{\text {in }}, k_{\text {out }}}$ is a cobordism between $\sqcup^{k_{\text {in }}} S^{1}$ and $\sqcup^{k_{\text {out }}} S^{1}$, i.e., $\Sigma_{g, k_{\text {in }}, k_{\text {out }}}: \sqcup^{k_{\text {in }}} S^{1} \Rightarrow \sqcup^{k_{\text {out }}} S^{1}$.

Let us next consider an oriented cobordism $M: m_{1} \Rightarrow m_{2}$. Pictorially, we can slice it somewhere in the middle, making sure that the slice is along an out-boundary, and thus find a decomposition of the original cobordism into two cobordisms $M_{1}: m_{1} \Rightarrow m^{\prime}$ and $M_{2}: m^{\prime} \Rightarrow m_{2}$, see figure 3 .

In particular, for the cylinder, we find

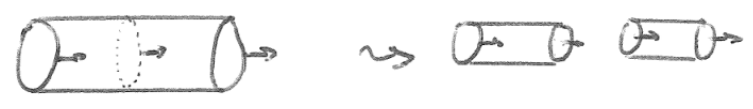

A different decomposition of the cylinder is presented in figure 4a. It gives rise to the snake decomposition, see figure $4 \mathrm{~b}$.

\section{Axiomatic topological quantum field theory}

In a Hamiltonian treatment of quantum field theory, we associate Hilbert spaces of states to constant "time", spatial slices, and we employ a time evolution operator (constructed in the usual manner from the Hamiltonian operator) to evolve states forward in time through spacetime. In standard quantum field theory on $\mathbb{R}^{n}$, the constant "time" slices are $\mathbb{R}^{n-1}$ and the Hamiltonian generates time-translations. In radial quantization of a conformal field theory, on the other hand, the constant "time" slices are spheres $S^{n-1}$ centered at the origin and the Hamiltonian generates dilatations. In topological quantum field theory, the spatial slices are $(n-1)$-dimensional manifolds. To each of them, we assign a state space. What's more, the Hamiltonian is zero, so no real dynamics takes place when evolving along a cylinder. However, non-trivial propagation does occur if the intervening manifold has nontrivial topology (and will only depend on that topology). In other words, the full spacetime is an $n$-dimensional manifold and time evolution is described by that manifold. 


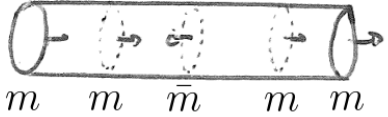

(a)

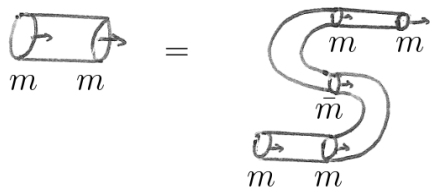

(b)

Figure 4: The snake decomposition of the cylinder.

\subsection{Definition of axiomatic TQFT}

These considerations lead us to the definition of an $n$-dimensional topological quantum field theory as introduced by Atiyah [1]. It is a rule $Z$ which

i) assigns to each $(n-1)$-dimensional, closed, oriented manifold $m$ a vector space $Z(m)$

ii) assigns to each oriented cobordism $M: m_{1} \Rightarrow m_{2}$ a linear map $Z(M): Z\left(m_{1}\right) \rightarrow Z\left(m_{2}\right)$ such that the following axioms hold:

A1: equivalent cobordisms must have the same images, i.e., if $M$ is homeomorphic to $M^{\prime}$ then $Z(M)=Z\left(M^{\prime}\right)$

A2: the cylinder $m \times I: m \Rightarrow m$ maps to the identity map, i.e., $Z(m \times I)=\mathrm{id}_{Z(m)}$

A3: if a cobordism $M$ can be decomposed as $M=M^{\prime} M^{\prime \prime}$ then $Z(M)=Z\left(M^{\prime \prime}\right) \circ Z\left(M^{\prime}\right)$ (composition of linear maps)

A4: disjoint unions map to tensor products

- if $m=m_{1} \sqcup m_{2}$, then $Z(m)=Z\left(m_{1}\right) \otimes Z\left(m_{2}\right)$

- if a cobordism $M: m_{1} \Rightarrow m_{2}$ is the disjoint union of $M^{\prime}: m_{1}^{\prime} \Rightarrow m_{2}^{\prime}$ and $M^{\prime \prime}: m_{1}^{\prime \prime} \Rightarrow m_{2}^{\prime \prime}$, then $Z(M)=Z\left(M^{\prime}\right) \otimes Z\left(M^{\prime \prime}\right)$

A5: empty manifolds $m=\emptyset$ map to the ground field $\mathbb{K}$, which we will take to be simply $\mathbb{C}$ in these lectures. An immediate consequence is that the cylinder over the empty manifold is the identity map on $\mathbb{K}$

Note that the first two axioms implement the topological nature of the theory.

\subsection{Some immediate consequences}

\subsubsection{Pairing and copairing}

Let $m$ be a closed $(n-1)$-manifold and let $V=Z(m)$ and $W=Z(\bar{m})$. Then we immediately find the existence of a pairing

$$
Z\left(\sum_{\substack{\tilde{m} \\ \sigma_{m}}}^{m}\right) \equiv \beta: V \otimes W \rightarrow \mathbb{K}
$$


and a copairing

$$
Z(\underbrace{C_{D} \rightarrow}_{\bar{m}}) \equiv \gamma: \mathbb{K} \rightarrow W \otimes V .
$$

Let us reconsider the snake decomposition of the cylinder, see figure 4b. It states that

$$
V \stackrel{\mathrm{id}_{V}}{\longrightarrow} V \stackrel{A 3}{=} V \stackrel{\mathrm{id}_{V} \otimes \gamma}{\longrightarrow} V \otimes W \otimes V \stackrel{\beta \otimes \mathrm{id}_{V}}{\longrightarrow} V,
$$

where we also used axiom A4. Let's denote $\beta(v, w)=\langle w \mid v\rangle$, and let's specify the copairing $\gamma$ by $\gamma\left(1_{\mathbb{K}}\right)=\sum_{i=1}^{n} w_{i} \otimes v_{i}$, where $w_{i} \in W, v_{i} \in V$. The snake then acts on an element $x \in V$ as

$$
x \mapsto \sum_{i} x \otimes w_{i} \otimes v_{i} \mapsto \sum_{i}\left\langle w_{i} \mid x\right\rangle v_{i} .
$$

As the snake is a decomposition of the cylinder cobordism, we learn that $x=\sum_{i}\left\langle w_{i} \mid x\right\rangle v_{i}$. We thus conclude that the pairing $\beta$ is nondegenerate because if for all $w \in W,\langle w \mid x\rangle=0$ then the snake decomposition implies that $x=0$, and, similarly, using the snake decomposition bending in the other direction, that if for all $v \in V,\langle y \mid v\rangle=0$ then $y=0$. With this nondegenerate pairing, we can identify $W=V^{*}$.

\subsubsection{TQFTs and topological properties}

Topological quantum field theories allow us to compute topological invariants. Indeed, let $M$ be a closed $n$-manifold whose topological properties we are interested in. $M$ can be considered as a cobordism $M: \emptyset_{n-1} \Rightarrow \emptyset_{n-1}$. Acting with the rule $Z$, we find a linear map $Z(M): \mathbb{K} \rightarrow \mathbb{K}$. Linear maps from the ground field to itself are fully specified by the constant $Z(M)\left(1_{\mathbb{K}}\right)$. This constant is a topological invariant as per the axioms that $Z$ obeys, in particular axiom A1. It can be computed explicitly by cutting up the manifold $M$ in various ways and using axiom A3.

A simple example is the product manifold $M=m \times S^{1}$ for some $(n-1)$-manifold $m$. If $Z(m)=$ $V$, we immediately find by cutting $M$ in half:

$$
\mathbb{K} \stackrel{Z(M)}{\rightarrow} \mathbb{K}=\mathbb{K} \stackrel{\gamma}{\rightarrow} V^{*} \otimes V \stackrel{\beta}{\rightarrow} \mathbb{K}
$$

and thus $Z(M)\left(1_{\mathbb{K}}\right)=\operatorname{dim} V .{ }^{3}$ The dimension of the vector space assigned to the manifold $m$ is thus a topological invariant of $M=m \times S^{1}$.

\subsection{Two-dimensional TQFTs}

In a two-dimensional topological quantum field theory, we need to know the vector space $Z\left(S^{1}\right)=V$ and a rule that maps any cobordism to a linear map subject to axioms A1-A5. However, we know that we can cut up cobordisms and consider the relevant compositions (see A3). Therefore, we can try to decompose any cobordism in terms of a small set of cobordisms that generate, by composition, all cobordisms. If we know the corresponding linear maps of this generating set of cobordisms, we have all necessary information that defines the TQFT.

\footnotetext{
${ }^{3}$ Some more details of this computation are as follows. Let $e_{i}$ be a basis for $V$ and $f_{j}$ be a basis for $V^{*}$. Furthermore, let $\gamma: \mathbb{K} \rightarrow V^{*} \otimes V: 1_{\mathbb{K}} \mapsto \sum_{i, j} \gamma^{i j} f_{j} \otimes e_{i}$, and let $\beta: V \otimes V^{*} \rightarrow \mathbb{K}$ be defined by $\left\langle f_{j} \mid e_{i}\right\rangle=\beta_{i j}$. Then the snake decomposition tells us that for all $x=\sim_{i} \lambda_{i} e_{i}$ we have $\sum_{i} \lambda_{i} e_{i}=\sum_{i, k, l} \lambda_{i} \gamma^{l k} \beta_{i l} e_{k}$. Hence $\sum_{l} \beta_{i l} \gamma^{l k}=\delta_{i}^{k}$.
} 


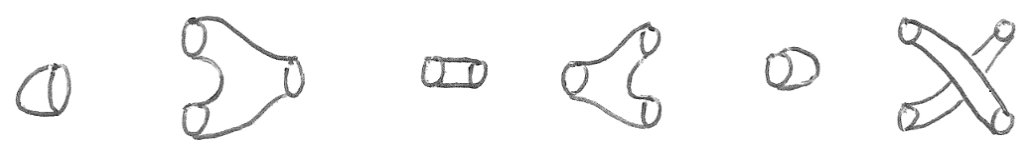

Figure 5: Generators of 2-cobordisms.

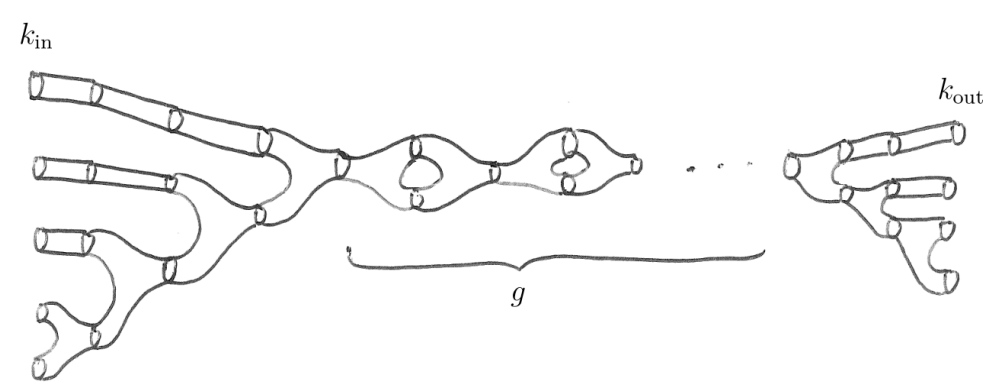

Figure 6: Pictorial proof that any 2-cobordism can be decomposed in terms of the generators of figure 5 .

\subsubsection{Generators of 2-cobordisms and their relations}

The full set of generators of 2-cobordisms is given in figure 5. We will assume symmetry of tensor-factors and further mostly ignore the last one. To prove that this is the full set of cobordisms, it is clearly sufficient to prove that any $\Sigma_{g ; k_{\mathrm{in}}, k_{\text {out }}}$ can be composed with these ingredients. Figure 6 makes clear that this is indeed the case. Note that when $k_{\text {in }}=0$ or $k_{\text {out }}=0$ we will need the cup and the cap.

The generators of figure 5 are not independent, but obey various relations. We give the list of relations in figures 7-9 (omitting various other relations that involve the crossed cylinders). The proof of this statement can be found, for example, in [8].

\subsubsection{Two-dimensional TQFT as Frobenius algebra}

Given the results of the previous subsection, our task when defining a two-dimensional TQFT is thus to assign linear maps to all the generators in figure 5 and to make sure that these maps satisfy the relations coming from the relations of these generators. Concretely, we have

$$
\begin{aligned}
& S^{1} \mapsto A \\
& S^{1} \sqcup S^{1} \sqcup \ldots \sqcup S^{1} \mapsto A^{n} \\
& \text { 凹ण } \mapsto\left[\mathrm{id}_{A}: A \rightarrow A\right] \\
& \mathcal{Q} \mapsto[\eta: \mathbb{K} \rightarrow A] \\
& \text { (D) } \mapsto[\varepsilon: A \rightarrow \mathbb{K}] \\
& \text { @ } \mapsto\left[\mu: A^{2} \rightarrow A\right]
\end{aligned}
$$

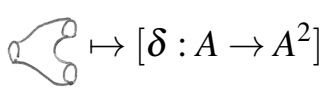




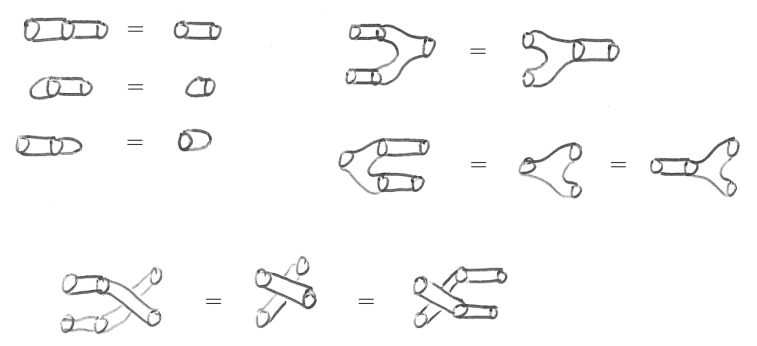

(a) Identity relations

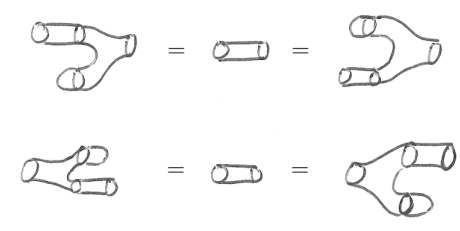

(b) Sewing in discs.

Figure 7: Relations among the generators of 2-cobordisms.

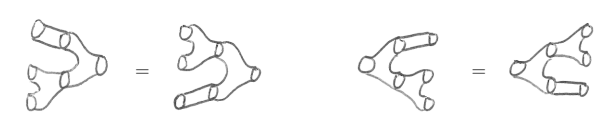

(a) (Co)-associativity

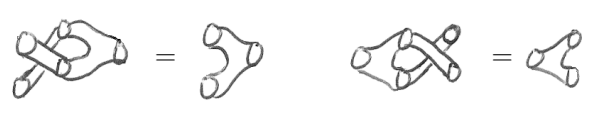

(b) (Co)-commutativity.

Figure 8: Relations among the generators of 2-cobordisms.

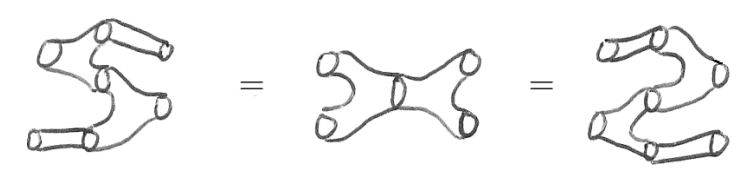

Figure 9: Frobenius relation among generators of 2-cobordisms.

A priori, A carries the structure of a vector space. However, the additional structure dictated by the images of the generators (satisfying the relations following from the images of figures 7-9) result in the algebraic structure of a Frobenius algebra. Note that in particular $\mu$ is simply the multiplication of the algebra. We have assumed it is commutative.

Example. Let us consider an explicit example. Let $A=\mathbb{K}[t] / t^{n}$, i.e., the polynomial ring in one variable modded out by the ideal generated by $t^{n}$. To make $\mathbb{K}[t] / t^{n}$ into a Frobenius algebra, we need to provide an additional datum, namely the so-called Frobenius form. It defines the image $\varepsilon$ of the cap. We take it to be $\varepsilon: A \rightarrow \mathbb{K}: t^{n-1} \mapsto 1$ and $t^{i} \mapsto 0$ for $i<n-1$. As for any two-dimensional topological field theory, the cylinder maps to the identity operator $i d_{A}: A \rightarrow A$. Furthermore, as mentioned above, $\mu: A^{2} \rightarrow A$ is the multiplication of $A$. Finally, we also know that the image of the cup (also known as the "unit") is $\eta: \mathbb{K} \rightarrow A: 1_{\mathbb{K}} \mapsto 1_{A}$.

Using the multiplication $\mu$ and the unit $\varepsilon$, we can construct the nondegenerate pairing $\beta$. Concretely, we take the basis of $A=\mathbb{K}[t] / t^{n}$ over $\mathbb{K}$ to be $1, t, t^{2}, \ldots, t^{n-1}$, and let us denote the basis of $A^{*}$ as $f_{t^{i}}, i=0, \ldots, n-1$. Then we find that $\left\langle f_{t^{i}} \mid t^{j}\right\rangle=\varepsilon\left(t^{i} t^{j}\right)=\varepsilon\left(t^{i+j}\right)=\delta_{i+j, n-1}$. Hence we can identify $f_{t^{i}} \simeq t^{n-1-i}$ and $A \simeq A^{*}$. The copairing $\gamma$ can be obtained as the dual of the pairing $\beta$. In our bases, it is given by $\gamma: \mathbb{K} \rightarrow A^{2}: 1_{\mathbb{K}} \mapsto \sum_{i} f_{t^{i}} \otimes t^{i}=\sum_{i} t^{n-1-i} \otimes t^{i}$. With these data, we can 
construct the comultiplication $\delta: A \rightarrow A^{2}$ as

$$
\delta: A \simeq A \otimes \mathbb{K} \stackrel{i d_{A} \otimes \gamma}{\longrightarrow} A \otimes A \otimes A \stackrel{\mu \otimes \mathrm{id}_{A}}{\longrightarrow} A \otimes A: t^{i} \mapsto t^{i} \otimes \sum_{j} t^{n-1-j} \otimes t^{j} \mapsto \sum_{j} t^{n-1+i-j} \otimes t^{j} .
$$

One can now explicitly verify that all relations of figures 7-9 are indeed satisfied.

Given the two-dimensional topological quantum field theory, we can compute topological invariants. For example, the two-sphere can be obtained by gluing the cup to the cap, i.e., $Z\left(S^{2}\right): \mathbb{K} \stackrel{\eta}{\rightarrow}$ $A \stackrel{\varepsilon}{\rightarrow} \mathbb{K}$. One then finds $\varepsilon\left(\eta\left(1_{\mathbb{K}}\right)\right)=\varepsilon\left(1_{A}\right)=0$. Similarly, by gluing the cup to a one-to-two pair of pants, whose two out-boundaries we glue in turn to a two-to-one pair of pants, which we finally cap off with a cap, we find for the torus $\varepsilon\left(\mu\left(\delta\left(\eta\left(1_{\mathbb{K}}\right)\right)\right)=\varepsilon\left(\mu\left(\delta\left(1_{\mathbb{A}}\right)\right)\right)=\sum_{j} \varepsilon\left(\mu\left(t^{n-1-j} \otimes t^{j}\right)\right)=\right.$ $\sum_{j} \varepsilon\left(t^{n-1}\right)=\sum_{j} 1=n$. This answer could of course have been more easily obtained as the dimension of the vector space $A$. By first computing the gluing of a one-to-two pair of pants to a two-to-one pair of pants in the form of the linear map $A \rightarrow A: t^{i} \mapsto n t^{n-1+i}$, one can easily convince oneself that all higher-genus surfaces have invariant 0 .

Example. A TQFT that produces more refined invariants is based on the algebra $A=\mathbb{K}[t] /\left(t^{n}-\right.$ 1 , with Frobenius form $\varepsilon: A \rightarrow \mathbb{K}: 1_{A} \mapsto 1_{\mathbb{K}}$ and $t^{i} \mapsto 0$ for $i>0$. Indeed, one finds that $Z\left(\Sigma_{g ; 0,0}\right)=$ $n^{g}$. This TQFT computes the genus.

\section{Schwarz- and Witten-type TQFTs}

Let us now develop the Lagrangian/path integral formulation of TQFT. We consider an $n$ dimensional, Riemannian manifold $M$ with a metric $g_{\mu \nu}$ and a quantum field theory defined on this space described by the action $S$. Let us further identify a collection of observables $\mathscr{O}_{\alpha}$ of the theory. Their correlation functions can be computed via the usual path integral

$$
\left\langle\mathscr{O}_{\alpha_{1}} \mathscr{O}_{\alpha_{2}} \ldots \mathscr{O}_{\alpha_{k}}\right\rangle=\int e^{-S} \mathscr{O}_{\alpha_{1}} \mathscr{O}_{\alpha_{2}} \ldots \mathscr{O}_{\alpha_{k}}
$$

The quantum field theory with (selected) observables $\left\{\mathscr{O}_{\alpha}\right\}$ is considered topological if

$$
\frac{\delta}{\delta g_{\mu v}}\left\langle\mathscr{O}_{\alpha_{1}} \mathscr{O}_{\alpha_{2}} \ldots \mathscr{O}_{\alpha_{k}}\right\rangle=0, \quad \forall k \geq 0, \forall \alpha_{i}, i=1, \ldots, k
$$

In other words, if the correlators of the $\left\{\mathscr{O}_{\alpha}\right\}$ are independent of the metric. Recall that we have argued before that on metric spaces topological invariants are metric independent.

\subsection{Schwarz-type topological quantum field theories}

To achieve the metric independence of (4.2), there are two options. The first option is realized in so-called Schwarz-type topological quantum field theories. These theories have the property that both the action and the observables are manifestly independent of the metric. Examples include 
- $B F$-theories, whose action is given by $S=\operatorname{tr} \int_{M} B \wedge F$, where $F$ is the Lie algebra-valued twoform field strength and $B$ is a Lie-algebra valued $n-2$-form. Metric-independent observables in this theory are standard Wilson loops $\operatorname{tr}_{R} P \exp \int_{\gamma} A$, where $\operatorname{tr}_{R}$ denotes the trace in the representation $R$ of the Lie algebra and $\gamma$ is a closed one-cycle.

An interesting example of this kind is two-dimensional Yang-Mills theory in the zero area limit. Let's put two-dimensional Yang-Mills theory on a surface $\Sigma$ with metric $g_{\mu v}$. The Yang-Mills action is given by $S_{\mathrm{YM}}=\frac{1}{4 e^{2}} \int_{\Sigma} d^{2} x \sqrt{g} \operatorname{tr} F^{2}$. Alternatively, it can be written as $S_{\mathrm{YM}}=\frac{1}{2} \int_{\Sigma}\left(i \operatorname{tr} \phi F+\frac{1}{2} e^{2} \operatorname{vol}(\Sigma) \operatorname{tr} \phi^{2}\right)$, where $\operatorname{vol}(\Sigma)$ is the volume form. Indeed, upon performing the Gaussian integral over the Lie algebra-valued scalar field $\phi$, we recover the original action. In the limit where the second term goes to zero, the theory becomes of the $B F$-type. If we do keep the second term, the theory does not have full general covariance, but it is invariant under area-preserving diffeomorphisms. One can straightforwardly develop the Hamiltonian picture of this theory and compute the image of the generators of two-cobordisms under $Z$. As the state space is infinite-dimensional, this theory (in the appropriate limit), while very similar, does not quite fit within the framework of axiomatic topological field theory developed above. An excellent set of lectures on two-dimensional Yang-Mills theory can be found in, for example, [6].

- Chern-Simons theory, which is described by the action $S=\operatorname{tr} \int_{M} A \wedge d A+\frac{2}{3} A \wedge A \wedge A$. Here $M$ is any three-manifold and $A$ is a gauge field. The theory's observables are again Wilson loops. A comprehensive set of lecture notes on Chern-Simons theory can be found in [9].

\subsection{Cohomological topological quantum field theories}

In these lectures we will focus on the second option, namely cohomological or Witten-type topological field theories. Let us assume that the quantum field theory possesses an infinitesimal symmetry generator, which we suggestively denote by $Q$. As $Q$ is a symmetry, we have $Q S=0$. Let us further assume that $Q$ is such that the observables are closed $Q \mathscr{O}_{\alpha}=0$ and the stress-energy tensor is exact, $\frac{\delta S}{\delta g^{\mu \nu}}=2 \sqrt{g} T_{\mu \nu}=Q K_{\mu \nu}$ for some symmetric tensor $K^{4}$. Then we can argue as follows:

$$
\begin{aligned}
\frac{\delta}{\delta g_{\mu \nu}}\left\langle\mathscr{O}_{\alpha_{1}} \mathscr{O}_{\alpha_{2}} \ldots \mathscr{O}_{\alpha_{k}}\right\rangle & =-\int \mathscr{O}_{\alpha_{1}} \mathscr{O}_{\alpha_{2}} \ldots \mathscr{O}_{\alpha_{k}} \frac{\delta S}{\delta g^{\mu \nu}} e^{-S} \\
& =-\int Q\left(\mathscr{O}_{\alpha_{1}} \mathscr{O}_{\alpha_{2}} \ldots \mathscr{O}_{\alpha_{k}} K_{\mu \nu} e^{-S}\right) \\
& =0
\end{aligned}
$$

where we assumed for simplicity that the observables are independent of the metric, but it clearly suffices that $\frac{\delta \mathscr{A}}{\delta g^{\mu \nu}}$ is exact for the argument to go through. Furthermore, we assumed that the path integral measure is invariant under $Q$, i.e., that $Q$ is non-anomalous. Note that it may be the case that the action is not just closed $Q S=0$, but also exact $S=Q \Lambda$. In this case, one can easily argue, similarly to the argument presented just now, that the correlation functions $\left\langle\mathscr{O}_{\alpha_{1}} \mathscr{O}_{\alpha_{2}} \ldots \mathscr{O}_{\alpha_{k}}\right\rangle$ will be independent of the coupling constant.

\footnotetext{
${ }^{4}$ Even though we are already employing cohomological terminology in stating that objects are "closed" or "exact", we do not necessarily impose that $Q^{2}=0$.
} 


\subsubsection{Topological twisting}

If we can find or construct quantum field theories with a symmetry $Q$ satisfying the above properties, a topological quantum field theory will emerge. A very widely applicable tool to construct precisely such theories was given in [4], and goes under the name of topological twisting. It can be employed in various dimensions and with various amounts of supersymmetry, but here we will focus on the original case of four-dimensional $\mathscr{N}=2$ super Yang-Mills theory. We start with a brief reminder of $\mathscr{N}=2$ supersymmetry.

The $\mathscr{N}=2$ Poincaré supersymmetry algebra contains eight supercharges organized as $Q_{\alpha}^{I}, \tilde{Q}_{\dot{\alpha} I}$. Here $\alpha, \dot{\alpha}$ are the usual $S U(2)_{1} \times S U(2)_{2}$ Lorentz indices, and $I$ is an $S U(2)_{R}$ R-symmetry index. The total R-symmetry is $S U(2)_{R} \times U(1)_{r}$. The supercharges $Q$ and $\tilde{Q}$ carry charges -1 and +1 under the abelian factor. In summary, the transformation rules of the supercharges under $S U(1)_{1} \times S U(2)_{2} \times S U(2)_{R} \times U(1)_{r}$ are

$$
\begin{aligned}
& Q:\left(\frac{1}{2}, 0, \frac{1}{2}\right)^{-1} \\
& \tilde{Q}:\left(0, \frac{1}{2}, \frac{1}{2}\right)^{+1}
\end{aligned}
$$

Their algebra is

$$
\left\{Q_{\alpha}^{I}, \tilde{Q}_{\dot{\alpha} J}\right\}=P_{\alpha \dot{\alpha}} \delta_{J}^{I}, \quad\left\{Q_{\alpha}^{I}, Q_{\beta}^{J}\right\}=\varepsilon_{\alpha \beta} \varepsilon^{I J} Z, \quad\left\{\tilde{Q}_{\dot{\alpha} I}, \tilde{Q}_{\dot{\beta} J}\right\}=\varepsilon_{\alpha \beta} \varepsilon_{I J} \bar{Z},
$$

where $P$ is the momentum generator and $Z$ the central charge. The latter won't be important for our discussion.

Note that, for example, $Q_{\alpha}^{1}, \tilde{Q}_{\dot{\alpha} 1}$ is an $\mathscr{N}=1$ subalgebra. The $\mathscr{N}=2$ vector multiplet can be decomposed in standard $\mathscr{N}=1$ multiplets as follows:

$$
(\mathscr{N}=2 \mathrm{VM})=(\mathscr{N}=1 \mathrm{VM} \oplus \mathscr{N}=1 \chi \mathrm{M} \text { in adjoint representation }),
$$

where VM stands for vector multiplet and $\chi \mathrm{M}$ for chiral multiplet. In total, the field content and its quantum numbers under $S U(1)_{1} \times S U(2)_{2} \times S U(2)_{R} \times U(1)_{r}$ is thus

$$
\begin{array}{cll}
\text { bosons: } & A_{\mu}: & \left(\frac{1}{2}, \frac{1}{2}, 0\right)^{0} \\
\phi, \tilde{\phi}: & (0,0,0)^{ \pm 2} \\
D_{(I J)}: & (0,0,1)^{0} \\
\text { fermions: } \lambda_{\alpha}^{I}, \tilde{\lambda}_{\dot{\alpha} I}: & \left(\frac{1}{2}, 0, \frac{1}{2}\right)^{+1} \oplus\left(0, \frac{1}{2}, \frac{1}{2}\right)^{-1} .
\end{array}
$$

Their transformation rules are given by

$$
\left\{\begin{array}{l}
\delta A_{\mu}=i\left(\xi^{I} \sigma_{\mu} \tilde{\lambda}_{I}\right)-i\left(\tilde{\xi}^{I} \tilde{\sigma}_{\mu} \lambda_{I}\right) \\
\delta \phi=-i\left(\xi^{I} \lambda_{I}\right) \\
\delta \tilde{\phi}=+i\left(\tilde{\xi}^{I} \tilde{\lambda}_{I}\right) \\
\delta \lambda_{I}=\frac{1}{2} \sigma^{\mu v} \xi_{I} F_{\mu v}+2\left(D_{\mu} \phi\right) \sigma^{\mu} \tilde{\xi}_{I}+2 i \xi_{I}[\phi, \tilde{\phi}]+D_{I J} \xi^{J} \\
\left.\delta \tilde{\lambda}_{I}=\frac{1}{2} \tilde{\sigma}^{\mu v} \tilde{\xi}_{I} F_{\mu v}\right)+2\left(D_{\mu} \tilde{\phi}\right) \tilde{\sigma}^{\mu} \xi_{I}+\tilde{\phi} \tilde{\sigma}^{\mu} D_{\mu} \xi_{I}-2 i \tilde{\xi}_{I}[\phi, \tilde{\phi}]+D_{I J} \tilde{\xi}^{J} \\
\delta D_{I J}=-\left(\tilde{\xi}_{I} \tilde{\sigma}^{\mu} D_{\mu} \lambda_{J}\right)+i\left(\xi_{I} \sigma^{\mu} D_{\mu} \tilde{\lambda}_{J}\right)-2\left[\phi,\left(\tilde{\xi}_{I} \tilde{\lambda}_{J}\right)\right]+2\left[\tilde{\phi},\left(\xi_{I} \lambda_{J}\right)\right]+(I \leftrightarrow J) .
\end{array}\right.
$$


Here $\xi_{\alpha}^{I}$ and $\tilde{\xi}_{\dot{\alpha} I}$ are constant supersymmetry parameters. The supersymmetric Yang-Mills action is given by

$$
\begin{aligned}
S_{\mathrm{YM}}=\frac{1}{g_{\mathrm{YM}}^{2}} \int d^{4} x \sqrt{g} \operatorname{tr} & {\left[\frac{1}{2} F_{\mu v} F^{\mu v}-\frac{1}{2} D^{I J} D_{I J}-4 D_{\mu} \tilde{\phi} D^{\mu} \phi+4[\phi, \tilde{\phi}]^{2}\right.} \\
& \left.-2 i\left(\lambda^{I} \sigma^{\mu} D_{\mu} \tilde{\lambda}_{I}\right)-2\left(\lambda^{I}\left[\tilde{\phi}, \lambda_{I}\right]\right)+2\left(\tilde{\lambda}^{I}\left[\phi, \tilde{\lambda}_{I}\right]\right)\right] .
\end{aligned}
$$

With these preparations, we are ready to implement Witten's topological twisting. The idea is simply to lock the $S U(2)_{2}$ and $S U(2)_{R}$ rotations. In other words, henceforth we consider $S U(2)_{2}^{\prime}=$ $\operatorname{diag}\left(S U(2)_{2}, S U(2)_{R}\right)$. The fields of the vector multiplet have the following transformation rules under the newly minted $S U(2)_{1} \times S U(2)_{2}^{\prime} \times U(1)_{r}$ symmetry group:

$$
\begin{aligned}
\text { bosons: } & \left(\frac{1}{2}, \frac{1}{2}\right)^{0} \text { still vector } \rightarrow A_{\mu} \\
& (0,0)^{ \pm 2} \text { still scalars } \rightarrow \phi, \bar{\phi} \\
& (0,1)^{0} \text { self-dual two-form } \rightarrow D_{\mu \nu}^{+} \\
\text {fermions: } & \left(\frac{1}{2}, \frac{1}{2}\right)^{+1} \text { vector } \rightarrow \psi_{\mu} \\
& (0,1)^{-1} \text { self-dual two-form } \rightarrow \chi_{\mu v}^{+} \\
& (0,0)^{-1} \text { scalar } \rightarrow \eta
\end{aligned}
$$

Here we introduced twisted fields transforming under the novel symmetry group. The supercharges themselves now transform as

$$
\left(\frac{1}{2}, \frac{1}{2}\right)^{-1} \oplus(0,1)^{+1} \oplus(0,0)^{+1}
$$

Note in particular the presence of a scalar supercharge, which we will call $Q$.

So far, on flat space, we haven't done anything really: we have just relabeled fields slightly unconventionally. However, it is an amazing fact that the supercharge $Q$ can be preserved on any spin-manifold (and in fact, more generally, on any manifold admitting a Spin $^{\mathbb{C}}$ structure). The Lagrangian we ought to consider is simply the covariantized flat-space Lagrangian with a certain $S U(2)_{R}$ background connection, proportional to the spin-connection, turned on. When the Lagrangian is written in terms of twisted fields, we don't have to actively worry about this background connection: the (spatial) index structure of the twisted fields suggests the proper LeviCivita connections in the covariant derivative. Concretely, one finds that the on-shell action (after some trivial rescalings of fields)

$$
\begin{aligned}
S=\int_{M_{4}} d^{4} x \sqrt{g} \operatorname{tr} & {\left[\frac{1}{4} F_{\mu v} F^{\mu v}+\frac{1}{2} \phi D_{\mu} D^{\mu} \bar{\phi}-i \eta D_{\mu} \psi^{\mu}+i\left(D_{\mu} \psi_{v}\right) \chi^{\mu v}-\frac{i}{8} \phi\left[\chi_{\mu v}, \chi^{\mu v}\right]\right.} \\
& \left.-\frac{i}{2} \bar{\phi}\left[\psi_{\mu}, \psi^{\mu}\right]-\frac{i}{2} \phi[\eta, \eta]-\frac{1}{8}[\phi, \bar{\phi}]^{2}\right]
\end{aligned}
$$

is invariant under the transformation rules

$$
\begin{aligned}
& \delta A_{\mu}=i \varepsilon \psi_{\mu}, \quad \delta \phi=0, \quad \delta \bar{\phi}=2 i \varepsilon \eta, \quad \delta \eta=\frac{1}{2} \varepsilon[\phi, \bar{\phi}], \\
& \delta \psi_{\mu}=-\varepsilon D_{\mu} \phi, \quad \delta \chi_{\mu \nu}=\varepsilon\left(F_{\mu \nu}+\frac{1}{2} \varepsilon_{\mu v \rho \sigma} F^{\rho \sigma}\right) .
\end{aligned}
$$


Note that both the action and the transformation rules of course still respect the $U(1)_{r}$ symmetry. It is easy to verify that the (on-shell) algebra is given by

$$
\left[\delta_{\varepsilon}, \delta_{\varepsilon^{\prime}}\right]=\operatorname{Gauge}(\rho)+\text { equations of motion },
$$

where $\rho=-2 i \varepsilon \varepsilon^{\prime} \phi$. Thus, on gauge invariant operators one has $Q^{2}=0$. Having identified a nilpotent supercharge for a class of quantum field theories we can place on any spin-manifold, we should still verify that the stress-energy tensor is $Q$-closed. A somewhat tedious and subtle computation confirms this fact

$$
T_{\mu \nu}=\left\{Q, \Lambda_{\mu \nu}\right\}, \quad \text { for } \quad \Lambda_{\mu \nu}=\frac{1}{2} \operatorname{tr}\left(F_{\mu \sigma} \chi_{\nu}{ }^{\sigma}+\ldots\right)
$$

What's more, one can also verify that

$$
\mathscr{L}^{\prime}=\mathscr{L}+\frac{1}{4} F_{\mu v} \tilde{F}^{\mu v}=\{Q, \Lambda\} \quad \text { for } \quad \Lambda=\frac{1}{4} \operatorname{tr} F_{\mu \nu} \chi^{\mu v}+\ldots
$$

We thus find ourselves in precisely the situation we described before. Hence, the partition function of the theory on a four-manifold $M_{4}$ computes a topological invariant of $M_{4}$, namely Donaldson's invariant.

Let us conclude by observing that there are various topological observables. First of all, from (4.24) we see that $Q \phi=0$. Hence all gauge-invariant combinations of $\phi$ are topological observables. In particular, for gauge group $S U(2)$, one has $W_{0}(P)=\frac{1}{2} \operatorname{tr} \phi^{2}(P)$ for some point $P \in M_{4}$. Note that

$$
\frac{\partial}{\partial x^{\mu}} W_{0}=\frac{1}{2} \frac{\partial}{\partial x^{\mu}} \operatorname{tr} \phi^{2}=\operatorname{tr} \phi D_{\mu} \phi=i\left\{Q, \operatorname{tr} \phi \psi_{\mu}\right\}
$$

so correlation functions of $W_{0}$ do not depend on their insertion points on $M_{4}$.

More observables can be constructed via topological descent. We start by rewriting the computation of (4.29) as

$$
0=i\left[Q, W_{0}\right], \quad d W_{0}=i\left\{Q, W_{1}\right\} \quad \text { with } \quad W_{1}=\operatorname{tr}\left(\phi \psi_{\mu}\right) d x^{\mu} .
$$

Then we continue by writing

$$
d W_{1}=i\left[Q, W_{2}\right], \quad d W_{2}=i\left\{Q, W_{3}\right\}, \quad d W_{3}=i\left[Q, W_{4}\right], \quad d W_{4}=0,
$$

with $W_{2}=\operatorname{tr}\left(\frac{1}{2} \psi \wedge \psi+i \phi F\right), W_{3}=i \operatorname{tr}(\psi \wedge F)$, and $W_{4}=-\frac{1}{2} \operatorname{tr} F \wedge F$. Given a $k$-dimensional homology cycle on $M_{4}$, we can consider

$$
I(\gamma)=\int_{\gamma} W_{k}, \quad \text { and } \quad[Q, I(\gamma)\}=\int_{\gamma}\left[Q, W_{k}\right\}=-i \int_{\gamma} d W_{k-1}=0,
$$

because $\gamma$ is a homology cycle. Inserting these additional topological observables gives rise to the higher Donaldson's invariants.

\section{Acknowledgments}

I would like to thank the organizers of the XV Modave Summer School in Mathematical Physics for inviting me to lecture. I'm grateful to Carlo Meneghelli and Yiwen Pan for helpful comments and useful discussions. My work is partially supported by grant \#494786 from the Simons Foundation. 


\section{References}

[1] M. F. Atiyah, Topological quantum field theory, Publications Mathématiques de l'IHÉS 68 (1988) 175.

[2] A. S. Schwarz, The partition function of degenerate quadratic functional and ray-singer invariants, Lett.Math.Phys. 2 (1978) 247.

[3] E. Witten, Quantum Field Theory and the Jones Polynomial, Commun. Math. Phys. 121 (1989) 351.

[4] E. Witten, Topological Quantum Field Theory, Commun. Math. Phys. 117 (1988) 353.

[5] D. Birmingham, M. Blau, M. Rakowski and G. Thompson, Topological field theory, Phys. Rept. 209 (1991) 129.

[6] S. Cordes, G. W. Moore and S. Ramgoolam, Lectures on 2-d Yang-Mills theory, equivariant cohomology and topological field theories, Nucl. Phys. Proc. Suppl. 41 (1995) 184 [hep-th/9411210].

[7] J. M. F. Labastida and C. Lozano, Lectures in topological quantum field theory, AIP Conf. Proc. 419 (1998) 54 [hep-th/9709192].

[8] J. Kock, Frobenius algebras and 2-d topological quantum field theories, vol. 59. Cambridge University Press, 2004.

[9] Gregory Moore, "Introduction to chern-simons theories." http://www.physics.rutgers.edu/ gmoore/TASI-ChernSimons-StudentNotes.pdf, 2019. 